\title{
The rich structure of transition in a shear flow
}

\author{
T. MULLIN
}

Manchester Centre for Nonlinear Dynamics, University of Manchester, Manchester M13 9PL, UK

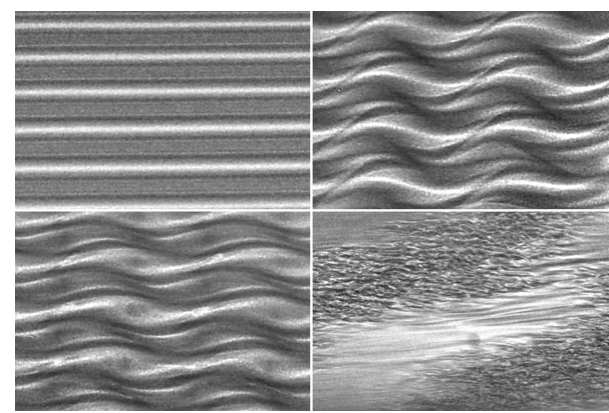

Significant advances have been made in applying ideas from nonlinear dynamical systems theory to flows which exhibit sequences of bifurcations in the transition to turbulence. Moreover, the recent discoveries of finite-amplitude states in linearly stable flows holds great promise for a breakthrough in our understanding transition in shear flows. Tsukahara, Tillmark \& Alfredsson (J. Fluid Mech., 2010, this issue, vol. 648, pp. 5-33) study a novel variant of a classical shear flow by adding global rotation. The competition between the induced body force and shear-induced instabilities leads to the discovery of a rich and beautiful tapestry of transition sequences.

\section{Introduction}

The prospect of gaining insights into turbulent motion by studying the stability of well-controlled specific flows has intrigued the fluid mechanics community for more than a century since the pioneering investigations of Rayleigh, Reynolds and Kelvin. Indeed Reynolds used this approach in his studies of pipe flow and anticipated that turbulence would set in gradually as the flow rate was increased. However, he was surprised to find that the onset of turbulence was abrupt and depended on the control of his experiment.

All theoretical and numerical evidence suggests that pipe flow is linearly stable and yet laboratory investigations demonstrate that turbulent flows are the norm even at modest Reynolds numbers, Re. Another much studied example of a linearly stable flow is in the plane Couette geometry where the fluid is driven by the differential motion of the walls. This flow has been shown to be nonlinearly stable for all values of $R e$ by Romanov (1973). It is fair to say that the transition to turbulence in both of the above problems remains largely mysterious despite a substantial amount of research.

A significant step forwards has been made in recent years in the understanding of such flows with the discovery of finite-amplitude states which can coexist with the linearly stable trivial laminar flow. These new states are disconnected from the trivial one and therefore cannot be reached with a continuous change in $R e$. However, they can be achieved by either a discontinuous jump in the control parameter or they can sometimes be reached by sequential continuous changes in more than one control parameter. Examples of finite-amplitude states are provided by Nagata (1990) in plane Couette flow, by Waleffe (2001) in channel flow, and in pipe flows by Faisst \& Eckhardt (2003) and Wedin \& Kerswell (2004). An idea in view is that the turbulent trajectories of the system decorate the fixed point structure of the finite-amplitude 
states and visit them with suitably weighted residence times as discussed by Willis \& Kerswell (2008). The discovery of these new states has brought fresh impetus and stimulated new research into problems where the origins of turbulence have remained unresolved since the studies of Reynolds and Couette.

A transition problem where considerable progress has been made is the flow between concentric cylinders commonly called Taylor-Couette flow. A sequence of bifurcations is found in the progression from laminar to disordered motion as $R e$ is increased and advances have been made by applying ideas from nonlinear dynamical systems and bifurcation theory. The flow is known to have rich structure in both the dynamical (Coles 1965) and static (Benjamin \& Mullin 1982) solution set and yet quantitative agreement between numerical calculations of the Navier-Stokes equations and experiment can be achieved (e.g. Mullin, Cliffe \& Pfister 1987).

Tsukahara, Tillmark \& Alfredsson (2010) report the results of a systematic experimental investigation into rotating Couette flow where both shear and body force instabilities arise. They have uncovered seventeen different flow regimes and a rich variety of transition scenarios. Multiple solutions and many routes to disordered motion are common features of closed recirculating flows but here they interestingly appear in an open flows where a single transition is most often assumed.

\section{Overview}

The experiments are performed in a carefully constructed water channel with walls which are made to move in opposite directions. The walls are made from a seamless transparent broad belt which is driven by a pair of cylinders and the entire apparatus is mounted on a rotating table to provide plane Couette flow with global rotation. Observations are made using flow visualization with anisotropic neutrally buoyant flakes which align with the local shear. Striking images of the various flow regimes are obtained and these are used to classify the various flow regimes in parameter space. Image processing is also performed to extract quantitative two point information from correlations of the light intensity which is used to provide estimates of the wavelength of the roll structures in the flow field.

The control parameters for the experiment are the global rotation number $\Omega$ and the Reynolds number $R e .\left(\Omega=2 \Omega_{z} h / v\right.$ and $R e=U_{w} h / v$ where $\Omega_{z}$ is the applied global rotation rate, $2 h$ is the gap width between the walls, $v$ is the kinematic viscosity and $2 U_{w}$ is the velocity difference of the walls.) These define the axes of a control parameter space and the protocols adopted mean that one-dimensional parameter sweeps are taken through the space. Moreover, smooth changes in parameter are made to circumvent possible multiplicity in the solution set with concomitant hysteresis. These procedures enabled estimates to be made for the parameter range of existence for the various flow states.

A prominent feature of the flows found for positive values of $\Omega$ is the striking similarity with flows observed in Taylor-Couette flows between rotating cylinders. Two of these flows have been highlighted in figure 1 where the simplest example of steady cellular flow can be compared directly in figures $1(a)$ and $1(c)$. The observed two-dimensional roll structures in the rotating plane Couette geometry are in accord with linear stability analysis with close agreement between the predicted and measured wavelengths. However, the subtlety of the boundary conditions in the physical realizations of rotary and rotating plane Couette flow may require further elucidation before a complete connection can be made. 


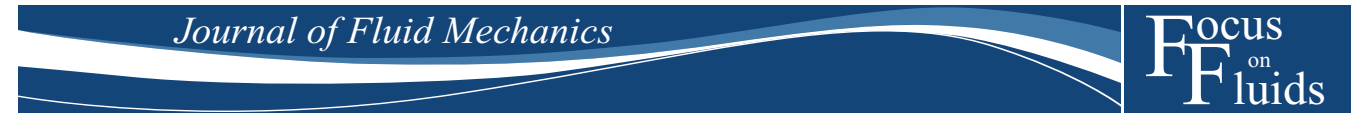

Taylor-Couette

Plane Couette with rotation
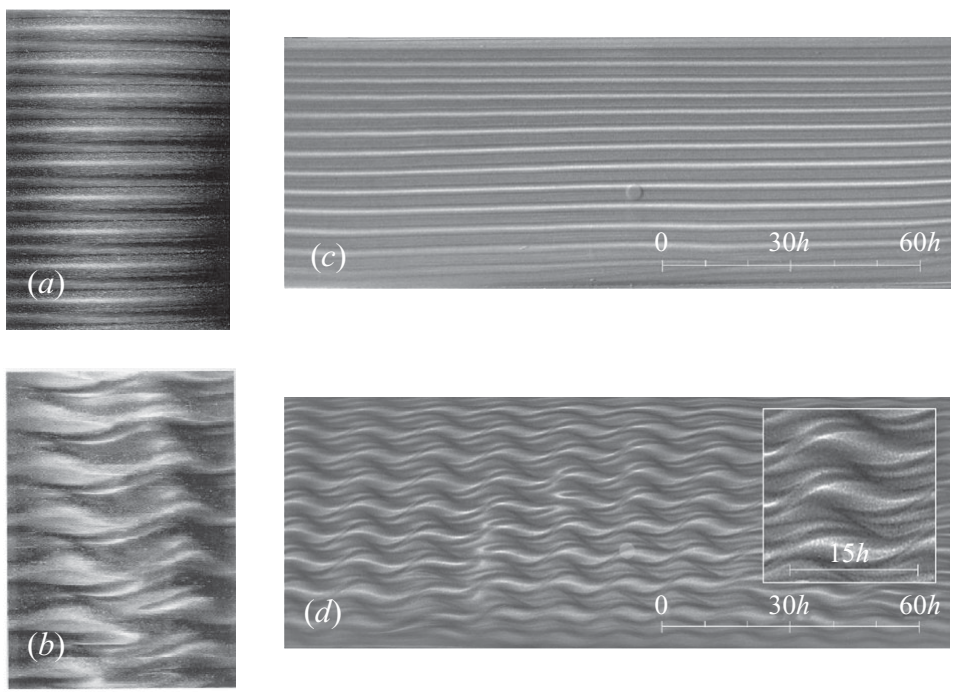

FIGURE 1. Taylor-Couette flows between concentric cylinders with aspect ratio $\Gamma=20$, radius ratio $\eta=0.5$. (a) $R e \approx 72.0(b) R e \approx 100$. Rotating plane Couette flow with $(c) \operatorname{Re}=178$, $\Omega=24.6$ and $(d) \operatorname{Re}=99.6, \Omega=8.65$. $(c)$ and $(d)$ are from Tsukahara et al. (2010).

The simply periodic travelling wave state for Taylor-Couette flow (figure $1 b$ ) is remarkably similar to the 'steady' three-dimensional cellular flow found in rotating plane Couette flow (figure $1 d$ ). The wave moves at $\sim 0.33$ the surface speed of the inner cylinder in Taylor-Couette flow and yet it is stationary in this new geometry. These flows concur with numerical calculations by Nagata (1998) who correctly predicts that they will be stable within a narrow parameter range.

Negative values of $\Omega$ stabilize the flow and the states comprise of localized regions of turbulent flow within a laminar background. These include turbulent stripes of the type first reported by Coles (1965) for counter-rotating cylinders and interesting turbulent 'spots' are found in a narrow wedge of parameter space. They are born and die at random locations and are visually reminiscent of Emmons spots (Emmons 1951) in boundary layer transition. Localized turbulent patches have also been observed in stationary plane Couette flow by Prigent \& Dauchot (2005).

\section{Future}

Hydrodynamic stability and the transition to turbulence is a vibrant research field where exciting theoretical developments such as the discovery of finite-amplitude solutions of the Navier-Stokes equations have been matched by high-quality observations of novel phenomena in classical flows. An example of this is provided by the fascinating range of flows uncovered in this careful experimental study where the apparently straightforward modification of adding rotation to a linearly stable shear flow has uncovered a plethora of transition sequences. Many of these are normally associated with internal recirculating flows where it might be assumed that they are 
special features of the geometry and hence have limited value in providing insight into open flows such as boundary layers and pipes.

A distinct advantage of problems such as Rayleigh-Bénard convection and TaylorCouette flow is that precisely controlled experiments can be used to produce highquality data which can provide quantitative comparison with numerical data on physical boundary conditions (e.g. Stevens et al. 2009). This aspect has enabled deep insights to be obtained into the origins and properties of turbulence under a wide range of parameters. Adopting a similar joint approach for open flows remains a formidable challenge from both experimental and numerical standpoints. An interesting prospect arising from the work of Tsukahara et al. (2010) is that it may be possible to form a bridge between the apparently distinct classes of open and closed flows by numerical and experimental parametric continuation.

An open and unresolved question is whether multiplicity found in experimental and numerical investigations at small values of $R e$ has relevance in turbulent flows, i.e. is the turbulent state in any given geometry unique? An interesting set of observations is provided by Monchaux et al. (2006) who find pairs of asymmetric states in a turbulent von Kármán flow. Is the complexity uncovered by Tsukahara et al. (2010) perhaps a generic feature of solutions of the Navier-Stokes equations?

\section{References}

Benjamin, T. B. \& Mullin, T. 1982 Notes on the multiplicity of flows in the Taylor experiment. J. Fluid Mech. 121, 219-230.

Coles, D. 1965 Transition in circular Couette flow. J. Fluid Mech. 21, 385-425.

Emmons, H. W. 1951 The laminar-turbulent transition in a boundary layer. Part 1. J. Aeronaut. Sci. 18, 490-498.

Faisst, H. \& EcKhardt, B. 2003 Travelling waves in pipe flow. Phys. Rev. Lett. 91, 224502.

Moncaux, R., Ravelet, F., Dubrulle, B., Chiffaudel, A. \& Daviaud, F. 2006 Properties of steady states in turbulent axisymmetric flows. Phys. Rev. Lett. 96, 124502.

Mullin, T., Cliffe, K. A. \& Pfister, G. 1987 Unusual time-dependent phenomena in TaylorCouette flow at moderately low Reynolds numbers. Phys. Rev. Lett. 58, 2212-2215.

Nagata, M. 1990 Three-dimensional finite-amplitude solutions in plane Couette flow; bifurcation from infinity. J. Fluid Mech. 217, 519-527.

NAGATA, M. 1998 Tertiary solutions and their stability in rotating plane Couette flow. J. Fluid Mech. 358, 357-378.

Prigent, A. \& Dauchot, O. 2005 Transition to versus from turbulence in subcritical Couette flows. In IUTAM Symposium on Laminar Turbulent Transition and Finite Amplitude Solutions (ed. T. Mullin \& R. R. Kerswell), Fluid Mechanics and its Applications, vol. 77, pp. 173-183. Springer.

Romanov, V. A. 1973 Stability of plane-parallel Couette flow. Funct. Anal. Appl. 7, 137-146.

Stevens, R. J. A. M., Zhong, J., Clercx, H. J. H., Ahlers, G. \& Lohse, D. 2009 Transitions between turbulent states in rotating Raleigh-Benard convection. Phys. Rev. Lett. 103, 024503.

Tsukahara, T., Tillmark, N. \& Alfredsson, P. H. 2010 Flow regimes in a plane Couette flow with system rotation. J. Fluid Mech. 648, 5-33.

WalefFe, F. 2001 Exact coherent structures in channel flow. J. Fluid Mech. 435, 93-102.

Wedin, H. \& Kerswell, R. R. 2004 Exact coherent structures in pipe flow: travelling wave solutions. J. Fluid Mech. 508, 333-371.

Willis, A. P. \& Kerswell, R. R. 2008 Coherent structures in localized and global pipe turbulence. Phys. Rev. Lett. 100, 124501. 